\title{
The rare BRAF VK600-601E mutation as a possible indicator of poor prognosis in rectal carcinoma - a report of a case
}

\author{
Yoshiko Mori ${ }^{1}$, Takeshi Nagasaka ${ }^{1 *}$, Hideyuki Mishima ${ }^{2}$, Yuzo Umeda ${ }^{1}$, Ryo Inada ${ }^{1}$, Hiroyuki Kishimoto ${ }^{1}$,
} Ajay Goel ${ }^{3}$ and Toshiyoshi Fujiwara ${ }^{1}$

\begin{abstract}
Background: The BRAF V600E mutation is reportedly associated with inferior survival among colon cancer patients. Here we report a patient with rectal cancer who carried the novel BRAF mutation VK600-601E, which has analogous molecular functions to those of the conventional BRAF mutation V600E, and may have potential as a prognostic marker for colorectal cancer (CRC).

Case presentation: The present 65-year-old male patient was diagnosed with recurrent rectal adenocarcinoma (stage II by AJCC TNM staging 7th edition) 14 months after surgery and was treated with modified FOLFOX6 (fluorouracil, leucovorin, and oxaliplatin), radiation, and FOLFIRI (fluorouracil, leucovorin, and irinotecan). The tumor progressed before further treatment could be initiated, resulting in death after 15 months. This survival period was similar to the median overall survival among patients with metastatic CRC and BRAF mutations who were treated with the FOLFIRI regimen with or without cetuximab.
\end{abstract}

Conclusions: Thus, the BRAF VK600-601E mutation may lead to an aggressive clinical course in CRC patients suffering from rapid progression and potential resistance to multiple therapeutic modalities.

Keywords: Rectal cancer, BRAF mutation, BRAF VK600-601E, Chemotherapy, Prognosis

\section{Background}

Prognoses for patients with colorectal cancer (CRC) have improved significantly with the introduction of moleculartargeted drugs such as anti-epidermal growth factor receptor agents (anti-EGFR). First-line treatment with the anti-EGFR cetuximab in addition to FOLFIRI (fluorouracil, leucovorin, and irinotecan) reduced the risk of progression of metastatic colorectal cancer compared with FOLFIRI treatment alone. However, the benefit of cetuximab was limited to patients with KRAS (codon 12 and 13) wild-type tumors [1]. S Tejpar et al. recently reported a number of candidate markers that influence the response to anti-EGFR, even among patients with wild type $K R A S$, including new KRAS mutations (codon 61 and 146) and mutations in BRAF and NRAS [2]. Although the best

\footnotetext{
* Correspondence: takeshin@cc.okayama-u.ac.jp

'Department of Gastroenterological Surgery, Okayama University Graduate School of Medicine Dentistry and Pharmaceutical Sciences, 2-5-1 Shikata-cho, Kita-ku, Okayama 700-8558, Japan

Full list of author information is available at the end of the article
}

characterized KRAS mutations on codons 12 and 13 are important, other KRAS mutations, such as those on codons 61 and 146, have received recent attention.

BRAF is a member of the RAF family of kinases and operates by binding to RAS [3]. A recent retrospective study of several clinical trials demonstrated that the presence of the BRAF V600E mutation was a strong prognostic factor for overall survival (OS) in patients with stage II/III CRC, particularly for tumors with low or stable microsatellite instability (MSI-Low, MSI-Stable, or no MSI) [4]. Activating mutations in the $B R A F$ gene are almost within the kinase domain and produce a signaling substitution of valine for glutamic acid at position 600 (V600E) [5]. However, in the present rectal cancer patient with wild-type $K R A S$ and no MSI, we discovered a novel $B R A F$ mutation that led to a triplet deletion of the coding nucleotides 1799-1801 (TGA1799-1801 deletion; VK600601E). This patient demonstrated relatively poor responses to conventional chemotherapy. Although this mutation 
has only been found in one patient to date, testing for this and other novel RAS mutations (KRAS codons 61 and 146 or $N R A S$ ) may provide essential prognostic markers that can be used to individualize treatment regimens for CRC patients.

\section{Case presentation}

A 65-year-old man presented with perineal pain, pollakiuria, and a serum carbohydrate antigen 19-9 level of $564.3 \mathrm{IU} / \mathrm{mL}$ (normal, <39.9 IU/mL). He had undergone a resection without preoperative chemoradiotherapy for a $4 \times 4.5-\mathrm{cm}$ rectal adenocarcinoma lesion 14 months previously, which was graded as IIA (T3NOM0) according to the AJCC TNM classification [6]. According to the surgical report, abdominoperineal resection with lateral lymph nodes resection and inferior mesenteric artery lymph node resection had done. Computed tomography (CT) and positron emission tomography (PET)/CT revealed a $3.5 \times 3.0 \times 2.7-\mathrm{cm}$ perineal metastatic lesion obstructing the right ureter. Metastases were observed in the right obturator lymph node, and the largest lesion measured $4.0 \times 3.3 \times 3.0 \mathrm{~cm}$. The metastatic lymph node had infiltrated the right internal iliac artery (Figure 1).

After stent placement in the ureter, four cycles of a modified FOLFOX6 regimen (mFOLFOX6) comprising $85 \mathrm{mg} / \mathrm{m}^{2}$ oxaliplatin, $200 \mathrm{mg} / \mathrm{m}^{2}$ leucovorin, $400 \mathrm{mg} / \mathrm{m}^{2}$ 5 -fluorouracil (5FU) bolus on day 1 and $2400 \mathrm{mg} / \mathrm{m}^{2} 5 \mathrm{FU}$ as a 46-h continuous infusion were administered at 2week intervals. Subsequently, the patient experienced lower back pain, and a second PET/CT examination indicated tumor recurrence and lymph node metastases on one side of the pelvis. Chemotherapy was terminated and radiotherapy (30 fractions at 2 Gy per day; total, 60 Gy) was initiated. Upon pain relief, mFOLFOX6 therapy was reinitiated according to the previous regimen, and no signs of disease progression were observed until 10 months later. At this point $\mathrm{PET} / \mathrm{CT}$ scans revealed that the perineal metastatic tumor had enlarged to approximately $9 \mathrm{~cm}$ (Figure 1), and the patient was hospitalized and treated with a FOLFIRI regimen comprising $150 \mathrm{mg} / \mathrm{m}^{2}$

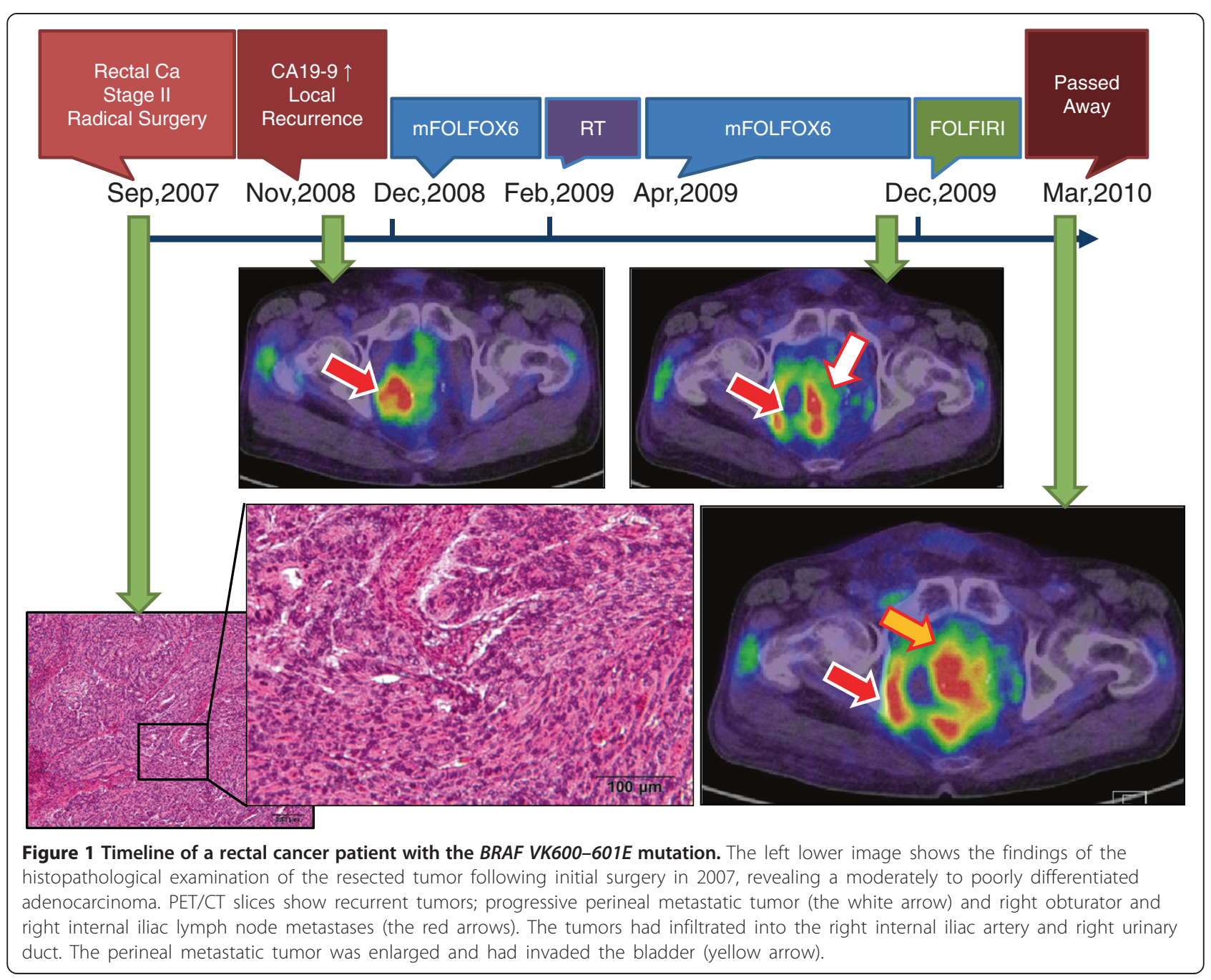


irinotecan, $200 \mathrm{mg} / \mathrm{m}^{2}$ leucovorin, $400 \mathrm{mg} / \mathrm{m}^{2} 5 \mathrm{FU}$ bolus on day 1 and $2400 \mathrm{mg} / \mathrm{m}^{2} 5 \mathrm{FU}$ as a $46-\mathrm{h}$ continuous infusion. One week later, the patient developed grade 4 neutropenia $\left(<500 / \mathrm{mm}^{3}\right.$; Common Terminology Criteria for Adverse Events ver. 4.0).

Between treatments, DNA was extracted from sections of the primary tumor tissue and analyzed for KRAS and $B R A F$ mutations and MSI [7]. No MSI or mutations at codons 12 or 13 of KRAS were identified. Direct sequencing for BRAF revealed a triplet nucleotide deletion (TGA) in coding nucleotides 1799-1801 (Figure 2). This mutation resulted in the deletion of amino acid 601 (lysine) and a valine-glutamate substitution at position 600 (VK600-601E).

After recovery from severe neutropenia, the perineal metastatic tumor had grown rapidly and had invaded the bladder (Figure 1), and consequent severe hematuria and nephropyelitis resulted in renal failure. Despite therapy for renal failure, the patient died 15 months after initiation of first-line therapy for tumor recurrence.

\section{Conclusions}

CRC development is considered a multistep process that follows the accumulation of genetic alterations, including chromosomal abnormalities, gene mutations, and epigenetic changes [4]. The Ras-Raf-MAP kinase pathway is known to mediate cellular responses to extracellular signals that regulate cell proliferation, differentiation, and apoptosis [8]. KRAS-activating mutations decrease or abolish the intrinsic GTPase activity of the KRAS protein, leading to its constitutive activation. Similarly, the BRAF V600E mutation induces structural changes that increase the kinase activity of the RAF protein [9]. Moreover, tumors with $R A S$ oncogene mutations are resistant to treatments with EGFR inhibitors, indicating that mutations in the RAS proto-oncogene are predictive of treatment responses $[4,10]$. RAS mutations commonly occur in codons 12 and 13 and are implicated in many human cancers, including approximately $40 \%$ of CRC cases [3]. In 2002, Davies et al. [5] identified activating mutations in BRAF that were present in many human cancers, including approximately $10 \%$ of CRC cases. The BRAF V600E mutation accounts for $80 \%$ of BRAF mutations in human cancers and is thought to be biologically distinct from less frequent $B R A F$ mutations because it allows growth in the absence of functional RAS genes [5]. Interestingly, the BRAF V600E mutation has not been previously reported in combination with the KRAS mutation in patients with CRC $[3,11]$, suggesting that at least one of these pathways must remain intact for cell survival.

The BRAF V600E mutation is known to be a strong prognostic marker in patients with metastatic and stage II/III CRC [4]. Similar to most activating mutations, the BRAF VK600-601E mutation affects the activation

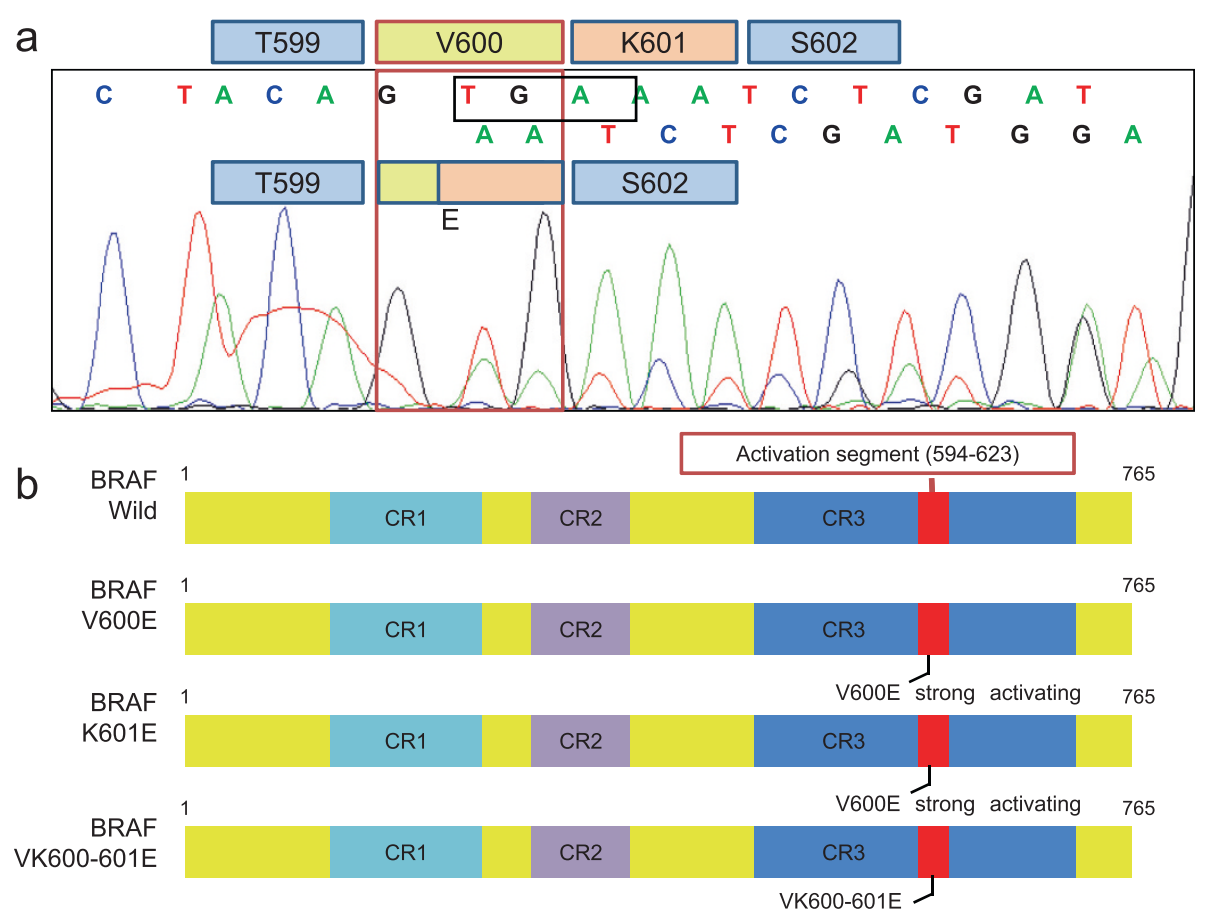

Figure 2 BRAF mutation analysis of the primary tumor. (a) Direct sequencing chromatographs of the BRAF VK600-601E mutation demonstrating triplet deletion from T1799 to A1801. (b) Schematic representation of the BRAF protein structure and various mutations. CR-1, -2 , and -3 represent conserved regions. The kinase activation segment is located between codons 594 and 623. Modified from Wan PT et al. [12]. 
segment of BRAF. Therefore, these mutations likely disrupt the interaction between the activation segment and P-loops that normally stabilize BRAF in the inactive conformation [12,13]. Because the BRAF VK600-601E mutation is functionally analogous to the $B R A F$ V600E mutation [14], it may be an additional marker of recurrence and poor treatment responses in patients with CRC. In support of this hypothesis, the present CRC patient responded poorly to conventional radiotherapy and chemotherapy and died at 15 months after initiation of first-line treatment for recurrence. This time period was almost equal to the median overall survival of patients with metastatic CRC with BRAF mutations who were treated with the FOLFIRI regimen with or without cetuximab (14.0 and 10.3 months, respectively) [15]. Although outcomes of rectal cancer often differ from those of colon cancer, clinical trials for unresectable advanced colorectal cancer always include colon and rectal cancer cases. For example, in a randomized phase III study (CRYSTAL trial) comparing FOLFIRI alone with cetuximab plus FOLFIRI regimens in 348 patients with KRAS exon2 wild-type CRCs, rectal cancer cases comprised $40.9 \%$ and $44.2 \%$ of the treatment arms, respectively [1].

To our knowledge, this is the first report describing the BRAF VK600-601E mutation in a patient with CRC. Although this mutation may be uncommon, the present observations warrant routine investigation of alternative mutations among CRC patients who show rapid progression and/or resistance to aggressive radiotherapy and/or chemotherapy.

\section{Consent}

This is a report of a case of standard treatment of the CRC with the rare somatic mutation. Written informed consent was obtained from the patient for testing the mutation status. Written informed consent for publication of this case report and accompanying images was obtained from a kin of the patient. However, we had not presented to our ethics committee, because this is not interventional study.

\section{Competing interests}

The authors declare that they have no competing interests.

\section{Authors' contributions}

YM carried out the genetic analyses and drafted the manuscript. TN treated the patient, participated in the genetic analyses and coordinated the manuscript. HM carried out the genetic analyses. YU treated the patient and collected patient's data. RI and HK carried out pathological staining and extracted DNA from Formalin-Fixed Paraffin-Embedded samples. AG and TF participated in the design of the study and drafted the discussion of the manuscript. All authors read and approved the final manuscript.

\section{Author details}

'Department of Gastroenterological Surgery, Okayama University Graduate School of Medicine Dentistry and Pharmaceutical Sciences, 2-5-1 Shikata-cho, Kita-ku, Okayama 700-8558, Japan. ${ }^{2}$ Cancer Center, Aichi Medical University, 1-1 Yazakokarimata, Nagakute City, Aichi 480-1195, Japan. ${ }^{3}$ Center of Gastrointestinal Research; and Center for Epigenomics, Cancer Prevention and Cancer Genomics, Baylor Research Institute and Charles A. Sammons Cancer Center, Baylor University Medical Center, 3500 Gaston Avenue, Suite H-250, Dallas, TX 75246, USA.

Received: 22 October 2014 Accepted: 7 January 2015

Published online: 31 January 2015

\section{References}

1. Van Cutsem E, Kohne CH, Hitre E, Zaluski J, Chang Chien CR, Makhson A, et al. Cetuximab and chemotherapy as initial treatment for metastatic colorectal cancer. N Engl J Med. 2009;360(14):1408-17.

2. Tejpar S, Piessevaux H. Personalized medicine in metastatic colorectal cancer treated with anti-epidermal growth factor receptor agents: a future opportunity? Asia Pac J Clin Oncol. 2014;10 Suppl 1:2-10.

3. Deschoolmeester $V$, Baay M, Specenier P, Lardon F, Vermorken JB. A review of the most promising biomarkers in colorectal cancer: one step closer to targeted therapy. Oncologist. 2010;15(7):699-731.

4. Roth AD, Tejpar S, Delorenzi M, Yan P, Fiocca R, Klingbiel D, et al. Prognostic role of KRAS and BRAF in stage II and III resected colon cancer: results of the translational study on the PETACC-3, EORTC 40993, SAKK 60-00 trial. J Clin Oncol. 2010;28(3):466-74.

5. Davies H, Bignell GR, Cox C, Stephens P, Edkins S, Clegg S, et al. Mutations of the BRAF gene in human cancer. Nature. 2002;417(6892):949-54.

6. Edge SB, Compton CC. The American joint committee on cancer: the 7th edition of the AJCC cancer staging manual and the future of TNM. Ann Surg Oncol. 2010;17(6):1471-4.

7. Nagasaka T, Koi M, Kloor M, Gebert J, Vilkin A, Nishida N, et al. Mutations in both KRAS and BRAF may contribute to the methylator phenotype in colon cancer. Gastroenterology. 2008;134(7):1950-60. 1960 e1951.

8. Peyssonnaux C, Eychene A. The Raf/MEK/ERK pathway: new concepts of activation. Biol Cell. 2001;93(1-2):53-62.

9. Loupakis F, Ruzzo A, Cremolini C, Vincenzi B, Salvatore L, Santini D, et al. KRAS codon 61, 146 and BRAF mutations predict resistance to cetuximab plus irinotecan in KRAS codon 12 and 13 wild-type metastatic colorectal cancer. Br J Cancer. 2009;101(4):715-21.

10. Peeters M, Price TJ, Cervantes A, Sobrero AF, Ducreux M, Hotko Y, et al. Randomized phase III study of panitumumab with fluorouracil, leucovorin, and irinotecan (FOLFIRI) compared with FOLFIRI alone as second-line treatment in patients with metastatic colorectal cancer. J Clin Oncol. 2010;28(31):4706-13

11. Nagasaka T, Sasamoto H, Notohara K, Cullings HM, Takeda M, Kimura K, et al. Colorectal cancer with mutation in BRAF, KRAS, and wild-type with respect to both oncogenes showing different patterns of DNA methylation. J Clin Oncol. 2004;22(22):4584-94.

12. Wan PT, Garnett MJ, Roe SM, Lee S, Niculescu-Duvaz D, Good VM, et al. Mechanism of activation of the RAF-ERK signaling pathway by oncogenic mutations of B-RAF. Cell. 2004;116(6):855-67.

13. De Falco V, Giannini R, Tamburrino A, Ugolini C, Lupi C, Puxeddu E, et al. Functional characterization of the novel T599I-VKSRdel BRAF mutation in a follicular variant papillary thyroid carcinoma. J Clin Endocrinol Metab. 2008:93(11):4398-402.

14. Hou P, Liu D, Xing M. Functional characterization of the T1799-1801del and A1799-1816ins BRAF mutations in papillary thyroid cancer. Cell Cycle. 2007;6(3):377-9.

15. Van Cutsem E, Kohne CH, Lang I, Folprecht G, Nowacki MP, Cascinu S, et al. Cetuximab plus irinotecan, fluorouracil, and leucovorin as first-line treatment for metastatic colorectal cancer: updated analysis of overall survival according to tumor KRAS and BRAF mutation status. J Clin Oncol. 2011;29(15):2011-9. 